\title{
CAUDAL BLOCK UNDER ULTRASOUND GUIDANCE
}

Jang Ho Roh, M.D., Dong Jin Chang, M.D., Jae Hoon Lee, M.D., Kyung Bong Yoon, M.D., Won Oak Kim, M.D., Duck Mi Yoon, M.D.

Department of Anesthesiology and Pain Medicine, Yonsei University College of Medicine, 134 Shinchon, Seodaemun-Gu, Seoul, Korea, 120-752

\section{INTRODUCTION}

Caudal block is useful when anesthesia for surgery or treatment of chronic pain is needed, but even when performed by an experienced physician has a failure rate of up to $25 \%,{ }^{1}$ due to improper needle placement. Herein, a study was undertaken to determine the success rate of caudal block, under ultrasound guidance, and the accuracy of the needle direction in the epidural space assessed. The anatomical structures in the sacral hiatus were also measured using ultrasound.

\section{METHODS}

After gaining the ethics committee approval, 46 patients undergoing a surgery or pain block received caudal blocks, which were performed after the anatomical structures in the sacral hiatus had been measured using ultrasound (Philips EnVisor HD linear probe). After skin infiltration by lidocaine, a 22-gauge, $6 \mathrm{~cm}$ needle was aseptically inserted toward the superior one-third of the sacrococcygeal ligament from the midsagittal view of the sacral hiatus using ultrasound. All these procedures were performed by the same anesthesiologist. The position and direction of the needle were identified using fluoroscopy by injecting a radio-opaque contrast through the needle. The time taken from the lidocaine injection to verification of the needle was measured and the planned nerve block then carried out.

\section{RESULTS}

All cases of needle insertion into the sacral canal, under ultrasound-guidance, were successful. The average duration of the procedure and trial count were $104.1 \pm 10.1$ seconds and $1.2 \pm 0.1$, respectively. In 10 of the 46 cases $(22 \%)$, the needle deviated either left or right in the sacral canal, so the direction of the needle had to be adjusted. The distance between two cornua, the depth of the sacral hiatus, and the thickness and length of the sacrococcygeal ligament were $17.1 \pm 0.4,3.9 \pm 0.3$, and $2.3 \pm 0.1$ and $24.9 \pm 0.9 \mathrm{~mm}$, respectively.

\section{CONCLUSIONS}

Ultrasound guidance can increase the success rate of the needle insertion into the sacral canal. However, even when ultrasound is used, the needle can deviate either left or right in the sacral canal.

\section{REFERENCE}

1. Anesthesiology $199991 ; 374-8$ 